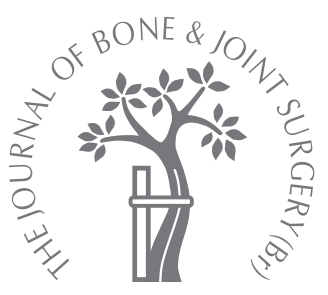

H.-C. Lim, J.-H. Bae, H.-R. Song, S. H. Teoh, H.-K. Kim, D.-H. Kum

From the Korea University College of Medicine, Seoul, Republic of Korea

RESEARCH

\title{
High tibial osteotomy using polycaprolactone-tricalcium phosphate polymer wedge in a micro pig model
}

\begin{abstract}
Medial open-wedge high tibial osteotomy has been gaining popularity in recent years, but adequate supporting material is required in the osteotomy gap for early weight-bearing and rapid union. The purpose of this study was to investigate whether the implantation of a polycaprolactone-tricalcium phosphate composite scaffold wedge would enhance healing of the osteotomy in a micro pig model. We carried out open-wedge high tibial osteotomies in 12 micro pigs aged from 12 to 16 months. A scaffold wedge was inserted into six of the osteotomies while the other six were left open. Bone healing was evaluated after three and six months using plain radiographs, CT scans, measurement of the bone mineral density and histological examination.

Complete bone union was obtained at six months in both groups. There was no collapse at the osteotomy site, loss of correction or failure of fixation in either group. Staining with haematoxylin and eosin demonstrated that there was infiltration of new bone tissue into the macropores and along the periphery of the implanted scaffold in the scaffold group. The CT scans and measurement of the bone mineral density showed that at six months specimens in the scaffold group had a higher bone mineral density than in the control group, although the implantation of the polycaprolactone-tricalcium phosphate composite scaffold wedge did not enhance healing of the osteotomy.
\end{abstract}

\footnotetext{
H.-C. Lim, MD, PhD,

Professor

H.-R. Song, MD, PhD,

Professor

H.-K. Kim, PhD, Student

Department of Orthopaedic

Surgery

Korea University Guro

Hospital, 80 Guro-Dong, Guro-

Gu, Seoul 152-703, Korea.

In.-H. Bae, MD, PhD, Assistant Professor

D.-H. Kum, MD, Resident

Department of Orthopaedic

Surgery

Korea University Ansan

Hospital, Gojan 1-Dong

Danwon Gu, Ansan-Si,

Gyeonggi-Do 425-707, Korea.

- S. H. Teoh, PhD, Professor

Department of Mechanical

Engineering

National University of

Singapore, 9 Engineering Drive

1, Singapore 117576.

Correspondence should be sent to Professor J.-H. Bae; e-mail: osman@korea.ac.kr
}

(C)2011 British Editorial Society of Bone and Joint Surgery doi:10.1302/0301-620X.93B1. $24767 \$ 2.00$

$J$ Bone Joint Surg [Br] 2011;93-B:120-5.

Received 2 March 2010;

Accepted after revision 17

August 2010
Recently, in order to try and overcome the disadvantages of autograft and allograft, ${ }^{1}$ attempts have been made to find adequate supporting material for augmentation after open-wedge high tibial osteotomy. Hydroxyapatite, ${ }^{2,3} \beta$ tricalcium phosphate ${ }^{4-7}$ or a combination of both ${ }^{8}$ are currently the most commonly used synthetic augments. Good clinical results have been reported with biocompatibility, absence of toxicity and non-inflammatory reactions, but relatively slow biodegradation and brittleness limit their application to load-bearing sites. ${ }^{2} \mathrm{~A}$ more recent approach is to use a composite of bioceramic and a resorbable polymer. ${ }^{9,10}$

Porous polymer scaffolds are promising materials for tissue engineering because they offer a physical, three-dimensional (3D) support and serve as a template for cell proliferation and ultimately the ingrowth of new tissue. Recently, polycaprolactone-based composite scaffolds fabricated by fused deposition modelling have been investigated, ${ }^{11-14}$ and shown to have a highly reproducible internal structure based on layerby-layer deposition of thermoplastic material. Such scaffolds were designed to retain their mechanical properties for five to six months and then gradually degrade over a period of two years. ${ }^{15}$ These scaffolds have a fully interconnected network of pores, which supports ubiquitous proliferation of cells ${ }^{16,17}$ while retaining their mechanical properties after implantation in experimental animal models. ${ }^{18-20}$

Rapid bone healing and early weight-bearing are clinically important, especially in patients with simultaneous bilateral open-wedge osteotomies. Many research teams have suggested that the use of supporting material could protect against collapse of the opening osteotomy and accelerate bony healing. However, to our knowledge, there are no reports which compare nonaugmented with augmented medial open-wedge high tibial osteotomy. The purpose of this study was to compare a non-augmented group with a polycaprolactone-based composite scaffold implantation group after open-wedge high tibial osteotomy in a micro pig animal model, in order to assess whether the implantation of a polycaprolactone-based composite scaffold would produce more rapid bone healing.

\section{Materials and Methods}

Scaffolds were fabricated with polycaprolactonetricalcium phosphate (ratio of composition $80: 20)$ microfilaments $(260 \mu \mathrm{m}$ to $370 \mu \mathrm{m})$ by 


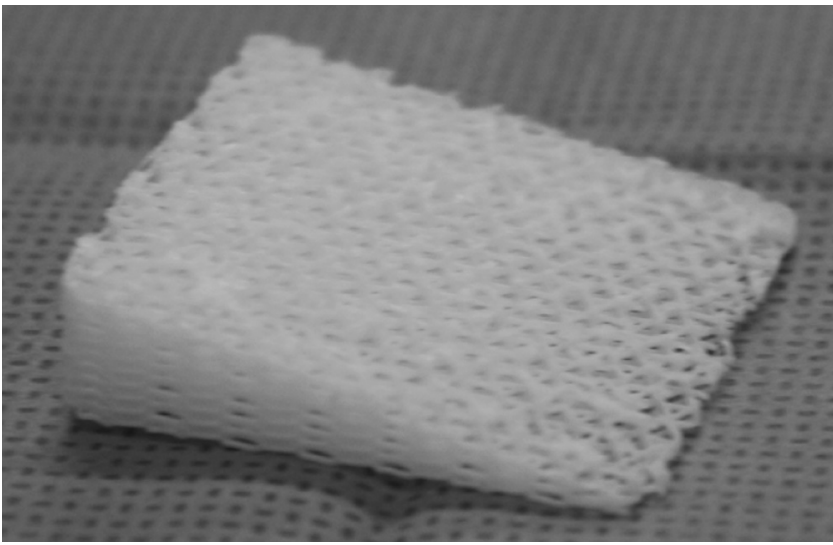

Fig. 1

Photograph showing the scaffold designed to fit the osteotomy site with a height of $9 \mathrm{~mm}$, length of $20 \mathrm{~mm}$ and width of $20 \mathrm{~mm}$.

fused deposition modelling as previously described. ${ }^{15,17,19,21}$ Fused deposition modelling, a rapid prototyping technology, was used to produce scaffolds with a honeycomb-like structure, and a fully interconnected network of channels, with controllable porosity and size of channel. The adhesion of each layer is achieved in a hydrostatic pressure chamber (from 0.01 to 10 atmospheres) by subjecting the block scaffold to a temperature just below the melting point of the polymer $\left(55^{\circ} \mathrm{C}\right.$ to $\left.60^{\circ} \mathrm{C}\right)$ for 30 to 45 minutes to allow proper sintering of each layer. Each scaffold manifests a lay-down pattern of $0 \% 60 \% 120^{\circ}$ with a porosity of $70 \%$ and average pore size of $0.515 \mathrm{~mm}$ as determined by micro-CT analysis. The scaffolds have a compressive modulus of $23.1 \mathrm{MPa}$ (SD 6.16) and a compressive strength of $6.38 \mathrm{MPa}$ (SD 0.82). A lay down pattern of $0 \% 60^{\circ} / 120^{\circ}$ was used to make a honeycomb array of interconnected equilateral triangles with a regular porous shape. In order to fit the osteotomy site, the scaffold was designed with a height of $9 \mathrm{~mm}$, a length of $20 \mathrm{~mm}$ and a width of $20 \mathrm{~mm}$ (Fig. 1).

The research protocol was approved by our institution. Medial open-wedge osteotomies were performed on the proximal tibia in 12 micro pigs (12 to 16 months old) weighing $30 \mathrm{~kg}$ to $50 \mathrm{~kg}$. The control group $(\mathrm{n}=6)$ had no augmented materials at the site of the osteotomy but the scaffold group $(\mathrm{n}=6)$ had a polycaprolactone-tricalcium phosphate scaffold wedge implanted into the opening gap (Fig. 2). At three months and six months after operation, three pigs in each group were killed and the osteotomies evaluated by CT scan, bone densitometry and and histological evaluation.

Operative procedure. All procedures were performed under general anaesthesia and aseptic conditions. An oblique skin incision $5 \mathrm{~cm}$ to $7 \mathrm{~cm}$ long, was made parallel to the upper border of the sartorius fascia. A guide pin was positioned $3 \mathrm{~cm}$ below the joint line at the level of the tibial tuberosity and advanced obliquely from the anteromedial aspect of the proximal tibia to $1 \mathrm{~cm}$ below the joint line at the posterolateral cortex. The osteotomy was created using a

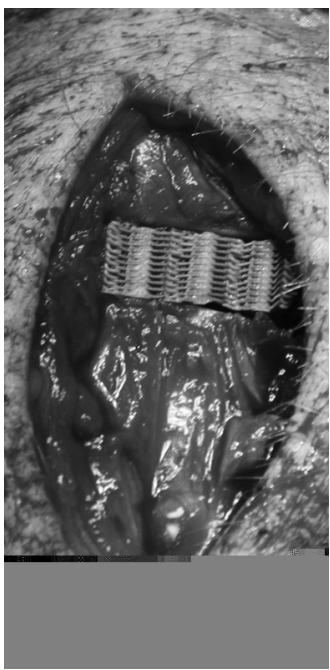

Fig. 2

Photograph showing the osteotomy surface after opening; the scaffold wedge has been implanted into the opening gap.

saw and osteotome, preserving the lateral cortex. The osteotomy surface was opened up and widened with a spreader. In the control group, a short $9 \mathrm{~mm}$ spacer plate was then placed at the anterior aspect of the superficial medial collateral ligament. The plate was fixed with a proximal and a distal screw. In the scaffold group, a custom-made polycaprolactone-tricalcium phosphate scaffold wedge was implanted into the gap. It was fashioned to be $9 \mathrm{~mm}$ high and $20 \mathrm{~mm}$ wide. Then a non-spacer plate was applied with a proximal and a distal screw. The soft tissues and skin were closed.

On the first post-operative day and at each month after operation, anteroposterior and lateral plain radiographs were taken under intravenous anaesthesia to assess the progression of bone union, as well as any loss of correction, failure of fixation or collapse of the osteotomy site. Nonunion was defined as no progression of bone bridging across the opening site at three or six months after operation with sclerotic margins at the periphery of the wedge. Loss of correction at the osteotomy site was determined by the change of the anatomical medial proximal tibia angle on the serial plain radiographs.

The CT density was measured to assess the formation of new bone at three and six months after operation. All CT images were acquired using a 64-channel multidetector row CT scanner (Brilliance 64; Philips Medical System, Cleveland, Ohio) with detector collimation of $16 \mathrm{~mm} \times 0.75 \mathrm{~mm}$, and tube energy and current of $120 \mathrm{kV}$ and $250 \mathrm{mAs}$, respectively, $0.7 \mathrm{~mm}$ beam pitch, and in the osteoscanning mode. The thickness of the display section was $2 / 1 \mathrm{~mm}$ (section thickness/section gap) and the image thickness for secondary raw data used in multiplanar reformatting was $1 / 0.5 \mathrm{~mm}$. 
Table I. Histological grade of healing of bone defect ${ }^{22}$

\begin{tabular}{ll}
\hline Grade & Histological findings \\
\hline 0 & No new bone \\
1 & New bone only at the inner edge of opening gap \\
2 & New bone at one-third of the opening gap \\
3 & New bone bridging between one-third and two-thirds of the opening gap \\
4 & New bone bridging the entire opening gap but not united \\
5 & Clearly united and remodelling \\
\hline
\end{tabular}

Table II. The ratio of mean CT density $(\mathrm{C} 1 / \mathrm{C} 2)^{*}$ in both groups

\begin{tabular}{lcll}
\hline & Three months & Six months & p-value \\
\hline Control group & 1.41 & 1.33 & 0.021 \\
Scaffold group & 1.39 & 1.11 & 0.013 \\
p-value & 0.05 & 0.037 & \\
\hline
\end{tabular}

${ }^{*} \mathrm{C} 1$, new bone formation area in the opening gap; $\mathrm{C} 2$, proximal metaphysis above the osteotomy site

Eight or nine coronal CT images were obtained for each specimen. An independent musculoskeletal radiologist measured the CT density value (Hounsfield Units) of the area of new bone formation $(\mathrm{C} 1)$ in the opening gap and in the proximal metaphysis (C2) above the osteotomy site using the Picture Archiving and Communications System (STARPACS; Infinitt Healthcare Co., Seoul, Korea). The average CT density value of $\mathrm{C} 1$ and $\mathrm{C} 2$ was calculated from all the coronal images in each specimen. The ratio of CT density of C1 to C2 was calculated and compared between the control and scaffold groups.

At three and six months after operation, the distribution of bone mineral density (BMD) was measured using dual energy x-ray absorptiometry (QDR 1000; Hologic, Bedford, Massachusetts). After scanning, the BMD of the two regions of interest was recorded; namely the area of new bone formation in the opening gap (R1) and the proximal metaphysis above the osteotomy site (R2). The ratio of the $\mathrm{BMD}$ value of $\mathrm{R} 1$ to $\mathrm{R} 2$ was calculated and compared between the control and scaffold groups.

Each specimen was fixed in $10 \%$ neutral-buffered formalin for two weeks and then processed for histology at three and six months after operation. Dehydration was accomplished using a graded series of ethyl alcohol (Harleco, Gibbstown, New Jersey) and cleared with xylene substitute (Thermo Electron Corporation, Waltham, Massachusetts). Infiltration was performed using a graded series of methylmethacrylate solutions (ACROS Organics, Morris Plains, New Jersey). Specimens were embedded using a catalysed mixture of Oste-Bed resin solution containing $2.5 \mathrm{~g}$ of benzoyl peroxide (Sigma-Aldrich, St Louis, Missouri) per $100 \mathrm{ml}$ under vacuum for at least 48 hours. After polymerisation, specimens were placed in a freezer for 24 hours. They were then trimmed of excess plastic (Isomet 1000; Buehler, Lake Bluff, Illinois), ground to expose the relevant portions (Ecomed 3; Buehler) and sectioned longitudinally
(3 $\mu \mathrm{m}$ to $4 \mu \mathrm{m}$ thick) using a microtome (Microm HM355S; Richard Allan Scientific, Kalamazoo, Michigan). Sections from each specimen were then stained with haematoxylin and eosin to evaluate new bone formation under a light microscope. The healing of regenerated bone was graded using a six point scale (Table I). ${ }^{22}$

Statistical analysis. The differences in the pixel values, CT density and BMD values between the two groups was assessed using the Mann-Whitney U test. Statistical analyses were performed using the SPSS (SPSS for Windows Release 15.0; SPSS Inc., Chicago, Illinois). A p-value $<0.05$ was considered statistically significant.

\section{Results}

Bone union was achieved at six months after operation in both groups. The trabecular continuity began at the inner portion of the osteotomy site and progression of trabecular continuity to the outer portion was observed in all specimens on serial plain radiographs. After operation the mean anatomical medial proximal tibial angle in the control group was $92^{\circ}$ (SD 2.4), and $94^{\circ}$ (SD 3.1) in the scaffold group. There was no significant change of angle at the final follow-up ( $p>0.05$ ) and valgus limb alignment was maintained well without loss of correction, failure of fixation or collapse of the osteotomy site in either group.

In both groups, partial bone formation was identified at the inner portion of the osteotomy site. The mean ratio of the CT density showed significant differences between the specimens at three and six-months in both the control and the scaffold groups $(\mathrm{p}=0.021$ and $\mathrm{p}=0.013$, respectively, Table II). At six months the specimens in the scaffold group showed higher levels of BMD ( $p=0.037$, Fig. 3 ).

The mean ratio of the BMD of new bone formation at three- and six-month specimens showed significant differences between the control and scaffold groups $(\mathrm{p}=0.033$ and $\mathrm{p}=0.045$, respectively, Table III). At six months the specimens showed a higher level of BMD in the scaffold group ( $\mathrm{p}=0.027$, Table III).

The histological grading was consistent with the CT density and the BMD (Table IV). New bone formation increased with time and the mean grade in the scaffold group was higher in the control group. In the control group, the defect in the gap was filled with mature bone and loosely organised fibrous tissue. The six-month specimens showed more extensive formation of new bone. In the 


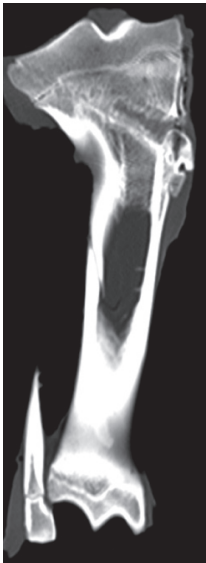

Fig. 3a

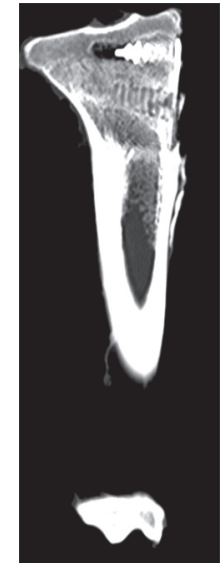

Fig. 3b
Coronal CT scan images showing six-month specimens from a) the control and b) the scaffold group. Bone formation is more evident in the scaffold group.

Table III. The ratio of mean bone mineral density $(\mathrm{R} 1 / \mathrm{R} 2)^{*}$ in both groups

\begin{tabular}{|c|c|c|c|}
\hline & Three months & Six months & p-value \\
\hline Control group & 0.69 & 0.81 & 0.033 \\
\hline Scaffold group & 0.62 & 0.91 & 0.045 \\
\hline$p$-value & $>0.05$ & 0.027 & \\
\hline
\end{tabular}

metaphysis above the osteotomy site

Table IV. The mean histological grading data

\begin{tabular}{|c|c|c|c|}
\hline & Three months & Six months & p-value \\
\hline Control group & 2.5 & 3.2 & 0.031 \\
\hline Scaffold group & 2.9 & 4.1 & 0.035 \\
\hline $\mathrm{p}$-value & $>0.05$ & 0.021 & \\
\hline
\end{tabular}

scaffold group, there was new bone formation within the interconnected pores of the scaffold and bone ingrowth from the host bone along the periphery of the scaffold (Fig. 4). There was no inflammatory reaction in either group.

\section{Discussion}

We wished to determine whether implantation of a polycaprolactone-based composite scaffold would produce faster bony consolidation compared with a non-augmented control group. Our CT scan and histological findings showed a higher level of BMD in the polycaprolactonetricalcium phosphate scaffold group six months after openwedge high tibial osteotomy. However, augmentation with a polycaprolactone-tricalcium phosphate scaffold wedge

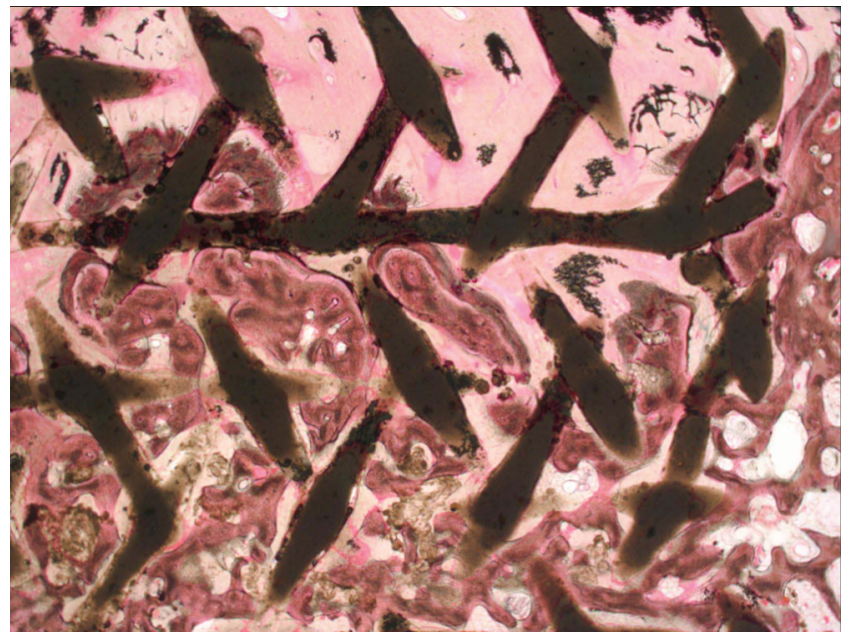

Fig. $4 \mathrm{a}$

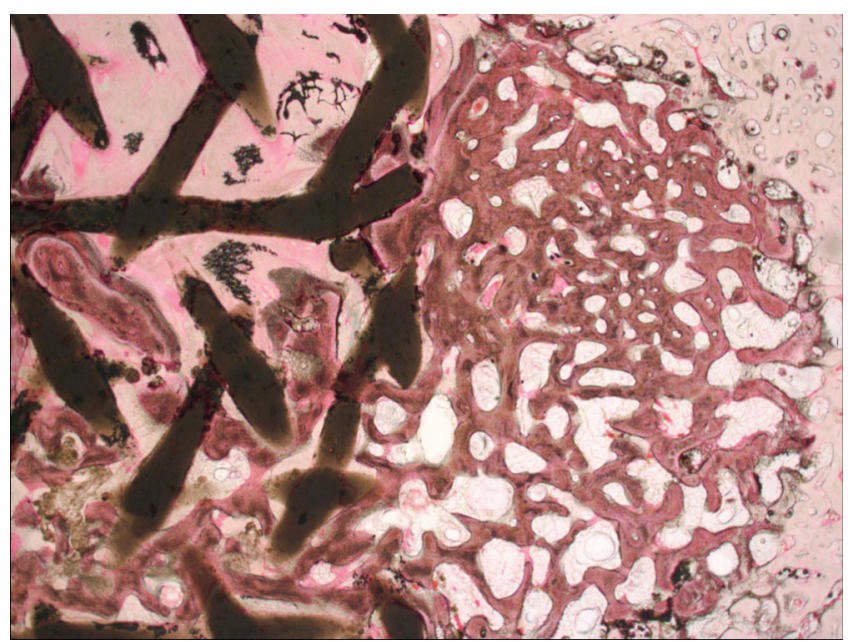

Fig. 4b

Photomicrographs (haematoxylin and eosin, $\times 1.25$ ) of a six-month specimen from the scaffold group showing a) new bone formation within the interconnected pores between the equilateral rods of scaffold (dark brown) and $\mathrm{b}$ ) bone ingrowth into the scaffold from the adjacent host bone.

did not show faster bony consolidation when compared with the non-augmented group.

In the context of proximal open-wedge tibial osteotomy, the limited availability of adequate autologous bone, and the morbidity associated with its harvest, has encouraged a search for appropriate substitute materials. Recent studies have reported that the use of hydroxyapatite alone or in combination with tricalcium phosphate is satisfactory. ${ }^{2-4,6}$ In these studies the histological and radiological findings showed a good resorption of the supporting material with complete incorporation and remodelling into new bone with increasing bony consolidation. However, there were no unaugmented control groups for comparison. One clinical study ${ }^{23}$ demonstrated that hydroxyapatite/tricalcium phosphate 
scaffolds promoted ingrowth of bone but showed no faster bony consolidation or any other additional benefits compared with the non-augmented osteotomy site with an opening angle $<10^{\circ}$. Likewise we also found that the implantation of polycaprolactone-tricalcium phosphate scaffold had no additional beneficial effects on bony consolidation when compared with a non-augmented group using a $9 \mathrm{~mm}$ bone defect in an animal model.

Most surgeons do not perform a bone graft in proximal tibial osteotomies with a gap $<10 \mathrm{~mm}$, because they believe that it is unnecessary. However, the rapid healing and early weight-bearing are clinically important, especially in patients with simultaneous bilateral osteotomy, even though the osteotomy gap may be small.

The scaffold used in the present study had a honeycomb array of interconnected equilateral triangles with a lay down pattern or $0 \% 60^{\circ} / 120^{\circ}$ and a porosity of $70 \%$. The constructs exhibited bone formation, remodelling and maturation along with rapid vascularisation in vivo. ${ }^{17,18,24,25}$ This scaffold architecture seemed to have favoured bone maturation and remodelling. ${ }^{17,24}$ However, differences could not be found between two groups, but an increased level of BMD was observed at six months in the scaffold group. This fully interconnected architecture was filled in all scaffolds by new bone formation and bone ingrowth from host bone.

We observed that the polycaprolactone-tricalcium phosphate scaffold remained in all specimens six months after operation. It has been found that the degradation of polycaprolactone is remarkably slow, requiring three years for complete removal from the host body. ${ }^{25}$ The addition of tricalcium phosphate particles increases the surface area available for hydrolysis, which may accelerate degradation of the scaffold. It is important to match the degradation rate to the rate of deposition of mineralised matrix in order to maintain the structural integrity of the defect while still promoting the remodelling of bone tissue, which takes at least 10 to 15 months in vivo. Rapid degradation can limit its application in engineering the induction of new bone tissue, especially at loadbearing sites.

This study has several limitations. The different types of plates (non-spacer plate $v s$ spacer plate) between the two groups may have affected the results. The primary stability of fixation is important for complete bony consolidation. Clinically, a less rigid construct can result in nonunion and loss of correction. It is possible that the spacer plate provided more stability than a non-spacer plate. However, because of lack of biomechanical data, it is not known which construct was more stable in our animal model. The polycaprolactone-tricalcium phosphate scaffold gives additional mechanical strength to the osteotomy with a non-spacer plate construct.

The implantation of the polycaprolactone-tricalcium phosphate composite scaffold wedge did not enhance healing of the osteotomy or provide any other additional benefits compared with the non-augmented osteotomy group at six months after open-wedge high tibial osteotomy in a micro pig model. The clinical relevance of this implant will require further study.

The authors wish to thank Dr J. H. Hwang, Mr Y. J. Kim, Mr T. H. Kim, Mr K. W. Nam and Ms C. M. Park for their technical assistance, Dr J. S. Myung for radiological evaluation, PWG Corporation for providing the animals and the animal laboratory and B. Braun Korea for providing instruments for this study.

No benefits in any form have been received or will be received from a commercial party related directly or indirectly to the subject of this article.

\section{References}

1. Fu F, Christel P, Miller MD, Johnson DL. Graft selection for anterior cruciate ligament reconstruction. Instr Course Lect 2009;58:337-54.

2. Koshino T, Murase T, Saito T. Medial opening-wedge high tibial osteotomy with use of porous hydroxyapatite to treat medial compartment osteoarthritis of the knee. J Bone Joint Surg [Am] 2003;85-A:78-85

3. Koshino T, Murase T, Takagi T, Saito T. New bone formation around porous hydroxyapatite wedge implanted in opening wedge high tibial osteotomy in patients with osteoarthritis. Biomaterials 2001;22:1579-82

4. van Hemert WL, Willems K, Anderson PG, van Heerwaarden RJ, Wymenga AB. Tricalcium phosphate granules or rigid wedge preforms in open wedge high tibial osteotomy: a radiological study with a new evaluation system. Knee 2004;11:451-6.

5. Tanaka T, Kumagae Y, Saito $\mathbf{M}$, et al. Bone formation and resorption in patients after implantation of beta-tricalcium phosphate blocks with $60 \%$ and $75 \%$ porosity in opening-wedge high tibial osteotomy. J Biomed Mater Res B Appl Biomater 2008;86:453-9.

6. Gaasbeek RD, Toonen HG, Van Heerwaarden RJ, Buma P. Mechanism of bone incorporation of beta-TCP bone substitute in open wedge tibial osteotomy in patients. Biomaterials 2005:26:6713-19.

7. Kraal T, Mullender M, de Bruine JH, et al. Resorbability of rigid beta-tricalcium phosphate wedges in open-wedge high tibial osteotomy: a retrospective radiological study. Knee 2008;15:201-5.

8. Gutierres M, Dias AG, Lopes MA, et al. Opening wedge high tibial osteotomy using 3D biomodelling Bonelike macroporous structures: case report. J Mater Sci Mater Med 2007;18:2377-82

9. Patka P, den Otter G, de Groot K, Driessen A. Reconstruction of large bone defects with calcium phosphae ceramics: an experimental study. Neth J Surg 1985;37:38-44

10. Chang YS, Oka M, Nakamura T, Gu HO. Bone remodelling around implanted ceramics. J Biomed Mater Res 1996;30:117-24.

11. Rai B, Ho KH, Lei Y, et al. Polycaprolactone- $20 \%$ tricalcium phosphate scaffolds in combination with platelet-rich plasma for the treatment of critical-sized defects of the mandible: a pilot study. J Oral Maxillofac Surg 2007;65:2195-205.

12. Rai B, Oest ME, Dupont KM, et al. Combination of platelet-rich plasma with polycaprolactone-tricalcium phosphate scaffolds for segmental bone defect repair. $J$ Biomed Mater Res A 2007;81:888-99.

13. Rai B, Teoh SH, Ho KH, et al. The effect of rhBMP-2 on canine osteoblasts seeded onto 3D bioactive polycaprolactone scaffolds. Biomaterials 2004;25:5499-506.

14. Rai B, Teoh SH, Hutmacher DW, Cao T, Ho KH. Novel PCL-based honeycomb scaffolds as drug delivery systems for rhBMP-2. Biomaterials 2005;26:3739-48.

15. Zein I, Hutmacher DW, Tan KC, Teoh SH. Fused deposition modeling of novel scaffold architectures for tissue engineering applications. Biomaterials 2002;23:1169-85.

16. Endres M, Hutmacher DW, Salgado AJ, et al. Osteogenic induction of human bone marrow-derived mesenchymal progenitor cells in novel synthetic polymerhydrogel matrices. Tissue Eng 2003;9:689-702.

17. Hutmacher DW, Schantz T, Zein I, et al. Mechanical properties and cell cultural response of polycaprolactone scaffolds designed and fabricated via fused deposition modeling. J Biomed Mater Res 2001;55:203-16.

18. Rohner D, Hutmacher DW, Cheng TK, Oberhozer M, Hammer B. In vivo efficacy of bone-marrow-coated polycaprolactone scaffolds for the reconstruction of orbital defects in the pig. J Biomed Mater Res B Appl Biomater 2003;66:574-80.

19. Schantz JT, Hutmacher DW, Chim H, et al. Induction of ectopic bone formation by using human periosteal cells in combination with a novel scaffold technology. Cell Transplant 2002;11:125-38.

20. Schantz JT, Hutmacher DW, Lam CX, et al. Repair of calvarial defects with customised tissue-engineered bone grafts. II: evaluation of cellular efficiency and efficacy in vivo. Tissue Eng 2003;9(Suppl 1):127-39.

21. Cao T, Ho KH, Teoh SH. Scaffold design and in vitro study of osteochondral coculture in a three-dimensional porous polycaprolactone scaffold fabricated by fused deposition modeling. Tissue Eng 2003;9(Suppl 1):103-12. 
22. Allen HL, Wase A, Bear WT. Indomethacin and aspirin = effect of nonsteriodal antiinflammatory agents on the rate of fracture repair in the rat. Acta Orthop Scand 1980;51:595-600

23. Aryee S, Imhoff AB, Rose T, Tischer T. Do we need synthetic osteotomy augmentation materials for opening-wedge high tibial osteotomy. Biomaterials 2008;29:3497-502.
24. Hutmacher DW, Goh JC, Teoh SH. An introduction to biodegradable materials for tissue engineering applications. Ann Acad Med Singapore 2001;30:183-91.

25. Pitt CG, Gratzl M, Kimmel GL, Surles J, Schindler A. Aliphatic polyesters. II: the degradation of poly (DL-lactide), poly (epsilon-caprolactone), and their copolymers in vivo. Biomaterials 1981;2:215-20. 